\title{
FAPAS proficiency testing of aflatoxin analysis in food and feed using DOA-Aflatoxin ELISA test kit
}

\author{
Chinaphuti, A. \\ Post Harvest and Processing Research and Development Office, \\ Department of Agriculture \\ (Chatuchark, Bangkok 10900 Thailand)
}

\begin{abstract}
DOA-Aflatoxin ELISA Test Kit is an in-house (ELISA test) kit produced by Department of Agriculture (DOA) Ministry of Agriculture and Cooperatives. The test kit had been validated for their precision and accuracy for aflatoxin $B_{1}$ detection in agricultural commodities. However, proficiency testing of analysis using DOA-Aflatoxin ELISA test kit is an essential element of test kit quality assurance. The analysis performance could be compared the analytical results with those from other laboratories with different methods.

The test materials of animal feed, pistachio paste, peanut butter slurry, peanut and chilli powder were prepared and sent to our laboratory by FAPAS (Food Analysis Performance Assessment Scheme). The analysis was performed for aflatoxin $\mathrm{B}_{1}$ using DOA-Aflatoxin ELISA test kit. The satisfactory results of Zscore were obtained from the tests. Performance in a FAPAS proficiency testing, therefore is considered satisfactory if a participant's Z-score lies within the range of -2 and +2 .
\end{abstract}

Key words: aflatoxin, ELISA test kit, proficiency testing

\section{Introduction}

Aflatoxins are the toxic secondary metabolites produced by fungi and was recognized as a public health problem since the late 1950 . The most toxic aflatoxin is aflatoxin $B_{1}\left(A_{F} B_{1}\right)$ which is classified as a human carcinogen by the International Agency of Research in Cancer $(\text { IARC })^{1)}$. The measurement of the contamination of aflatoxin has been the subject of constant improvement of analytical technology. The techniques have involved Thin-Layer Chromatography (LC) and Enzyme Link Immunosorbent Assay (ELISA) ${ }^{2}$. However, many scientific societies are actively involved in development and validation of analytical method.

DOA-Aflatoxin ELISA Test Kit is an in-house test kit which was developed by Postharvest and Processing Research and Development Office, Department of Agriculture, Ministry of Agriculture and Cooperative, Thailand (Fig. 1). Principle of the test is based on a direct competitive enzyme-linked immunosorbent assay. The test kit was produced for qualitative and quantitative detection aflatoxin $B_{1}$ results in food and feed. There was no significant difference in detection efficiency of this test kit compared to traditional methods of minicolomn, TLC and HPLC. The kit was validated for their recovery from spike samples which obtained 82-100\% recovery ${ }^{3)}$. The DOA-Aflatoxin ELISA Test Kit was widely used in Thailand to screen the aflatoxin $B_{1}$ free raw materials prior to processing. Consequently, finished products of both food and feed are quiet safe from afratoxin contamination. 
To continue ensuring the quality of this in-house test kit, laboratory proficiency testing is an essential element. There are many mycotoxin check sample programs sponsored by a variety of agencies such as International Agency for Research on Cancer (IARC), the American Association of Feed Control Officials (AAFCO), the American Oil Society $(\text { AOCS })^{4)}$ and Food Analysis Performance Assessment Scheme (FAPAS). We did proficiency testing using DOA-Aflatoxin ELISA Test Kit as the analysis of an external quality check sample as part of laboratory's routine procedures with FAPAS.

\section{Materials and methods}

Test materials The test materials were prepared by laboratory contracted to do so by FAPAS. Test material of $55 \mathrm{~g}$ was packed in a padded envelop, together with a covering letter, electronic submission of result, method instruction and the result form were send to our laboratory by mail. The test materials were pistachio paste, peanut butter slurry, animal feed, peanut and chilli powder.

\section{Aflatoxin $B_{1}$ detection by DOA-Aflatoxin ELISA Test Kit}

Samples preparation $20 \mathrm{~g}$ of each test materials were weighted and combine with $100 \mathrm{~mL}$, of extraction solvent $(70 \% \mathrm{MeOH})$, which was homogenized by high speed blender for 2-3 minutes. The homogenate were allowed to stand for 5 minutes for setting of the slurry. The mixture was filtered to afford a minimum of $15 \mathrm{~mL}$ of the extract through a Whatman No.4 filter paper into a clean container. Prior to be subjected to ELISA , the filtrate was diluted to $1: 20$ with $0.01 \mathrm{M}$ phosphate buffer saline.

\section{Analysis procedures}

1. Add $50 \mu \mathrm{L}$ of each standard dilutions $(0,0.2,0.5,1$ and $2 \mathrm{ng} / \mathrm{mL})$ was added into the coated well (4-5 well for standard and the less for sample)

2. $50 \mu \mathrm{L}$ of enzyme conjugate was added into every well, then slightly shaked.

3. The mixture was incubated at room temperature for 30 minutes under dark condition.

4. The contents of the well were dumped into the appropriate waste container.

5. Wells were washed thoroughly 3-5 times with washing buffer, then taped out on absorbent paper towel.

6. $100 \mu \mathrm{L}$ TMB substrate with freshly prepare was transferred into the wells.

7. The walls were incubate at room temperature in the dark for 5 minutes.

8. $100 \mu \mathrm{L}$ stopping solution $(0.03 \mathrm{M}$ phosphoric acid) was added into each well.

9. The absorbance of each well at $450 \mathrm{~nm}$ was measured using microELISA reader.

Calculation Quantitative interpretation was based on standard curve established by the absorbance of standard aflatoxin in the test. Program log-logit/log was used for calculation of aflatoxin concentration. This value had to be multiplied by a factor(x20) to correct the result for dilution of the samples to obtain concentration of aflatoxin $B_{1}$ in the test samples. The results were submitted to FAPAS for statistical evaluation in comparison to other laboratories.

\section{Results and discussion}

The test materials used in this proficiency testing were pitachio paste, peanut butter slurry, two animal feed samples, peanut and chili powder. The results of a typical chemical analysis were normally distributed. The majority of results will be centered on a mean value but, inevitably, some results will lie at the extremes of the distribution. The statistics of a 
normal mean that about $95 \%$ of data points will lie between a Z-score of -2 and +2 . Performance in a FAPAS proficiency test, therefore, was considered 'satisfactory' if a participant ' $z$-score lies within this range. It follows that if a participant's z-score lied outside $|Z|>2$ there is about a 1 in 20 chances that their results was in fact an acceptable result from the extreme of the distribution. If participant's $\mathrm{Z}$-score lies outside $|\mathrm{Z}|>3$ chance that their results is actually acceptable is only about 1 in 300 .

Each participants was given a laboratory number, assigned in order to receipt of results. The results of aflatoxin $B_{1}$ dectected from the test materials in FAPAS proficiency testing using DOA-Aflatoxin ELISA Test Kit as the in-house method were 'satisfactory '(Table 1). $\mathrm{Z}$-score of six test materials were lied between -2 to 1.0 . The lowest $\mathrm{z}$-score of -0.1 and -0.3 were shown in pistachio paste test material whereas the highest Z-score of -2.0 was found in animal feed when DOA-Aflatoxin ELIZA Test Kit was used as routine and peanut butter slurry (Fig. 1,2) whereas the highest z-score of animal feed was -1.4 and -2 .

Each round of the test material, there were about 62-105 laboratories participated in proficiency testing. The analytical method used in most of the laboratories was HPLC, only 13 laboratories including our laboratory used ELISA method for aflatoxin $\mathrm{B}_{1}$ detection. The results from proficiency testing are one of several tools that enable participants to make that judgement themselves. Accreditation bodies will also ask laboratories for details of their performance. Hence, the satisfactory results of z-score which we obtained from FAPAS proficiency testing could be ensured the quality of DOA-Aflatoxin ELISA test kit used as analytical method for aflatoxin $\mathrm{B}_{1}$.

Table 1. Results of aflatoxin $B_{1}$ in FAPAS proficiency test materials detected by DOA-Aflatoxin ELISA test kit and their Z-scores.

\begin{tabular}{|l|c|c|c|}
\hline \multicolumn{1}{|c|}{ Test materials } & $\begin{array}{c}\mathrm{AFB}_{1}(\mu \mathrm{g} / \mathrm{kg}) \\
\text { (actual value) }\end{array}$ & $\begin{array}{c}\mathrm{AFB}_{1}(\mu \mathrm{g} / \mathrm{kg}) \\
\text { (assigned value) }\end{array}$ & Z-score \\
\hline Pistachio paste & 16.90 & 17.10 & -0.1 \\
Peanut butter slurry & 5.51 & 6.35 & -0.3 \\
Animal feed & 6.61 & 11.80 & -2.0 \\
Peanut & 5.51 & 6.35 & -0.6 \\
Chilli powder & 20.31 & 16.70 & 1.0 \\
Animal feed & 5.10 & 7.28 & -1.4 \\
\hline
\end{tabular}

FAPAS" Alatoxins Repar 0460

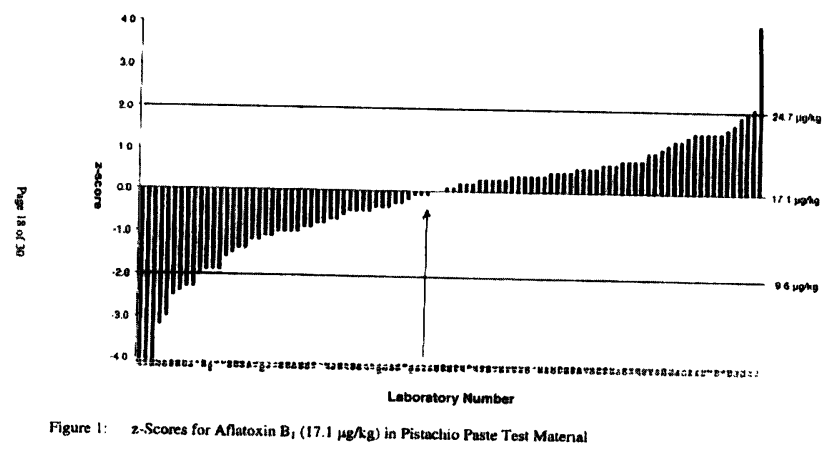

Fig. 1. Z-score for aflatoxin $B_{1}$ in pistachio paste test material 


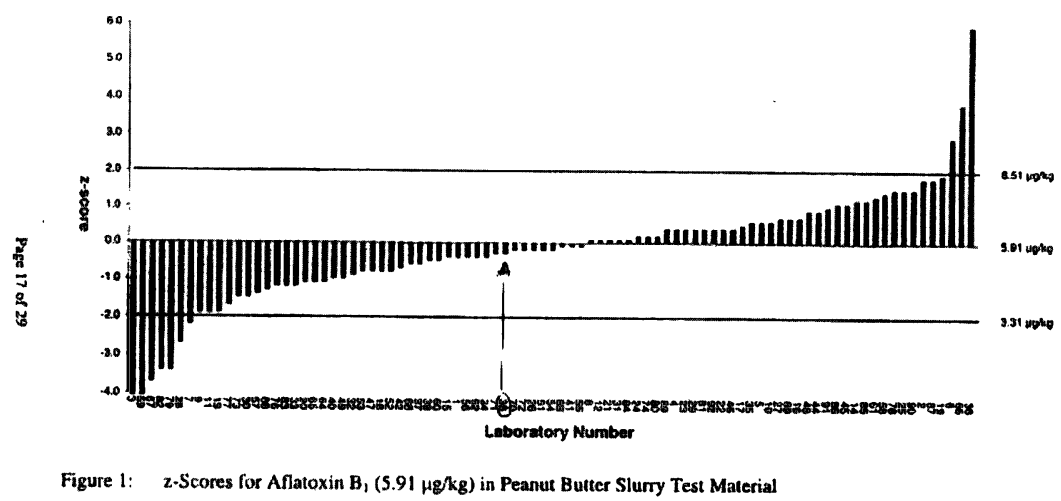

Fig. 2. Z-score for aflatoxin $B_{1}$ in peanut butter slurry test material

\section{References}

1) Moss, M.O: Mycological Research, 100, 513-523(1998)

2) Horwitz, W., Albert, R., Nesheim, S.: J.AOAC Int., 76, 461-491 (1993)

3) Chinaphuti, A., Trikarunasawat. C., Wongurai. A. Kositcharoenkul, S.: Kasetsart J. Nat. Sci., 36, 179-186 (2003)

4) Chu,F.S.: Mycotoxin Analysis. In.Analyzing Food for Nutrition Labeling and Hazardous Contaminants (1995) Eds by Jeon I.J., Ikins, W.G., Marcel Dekker, pp. 283-331 

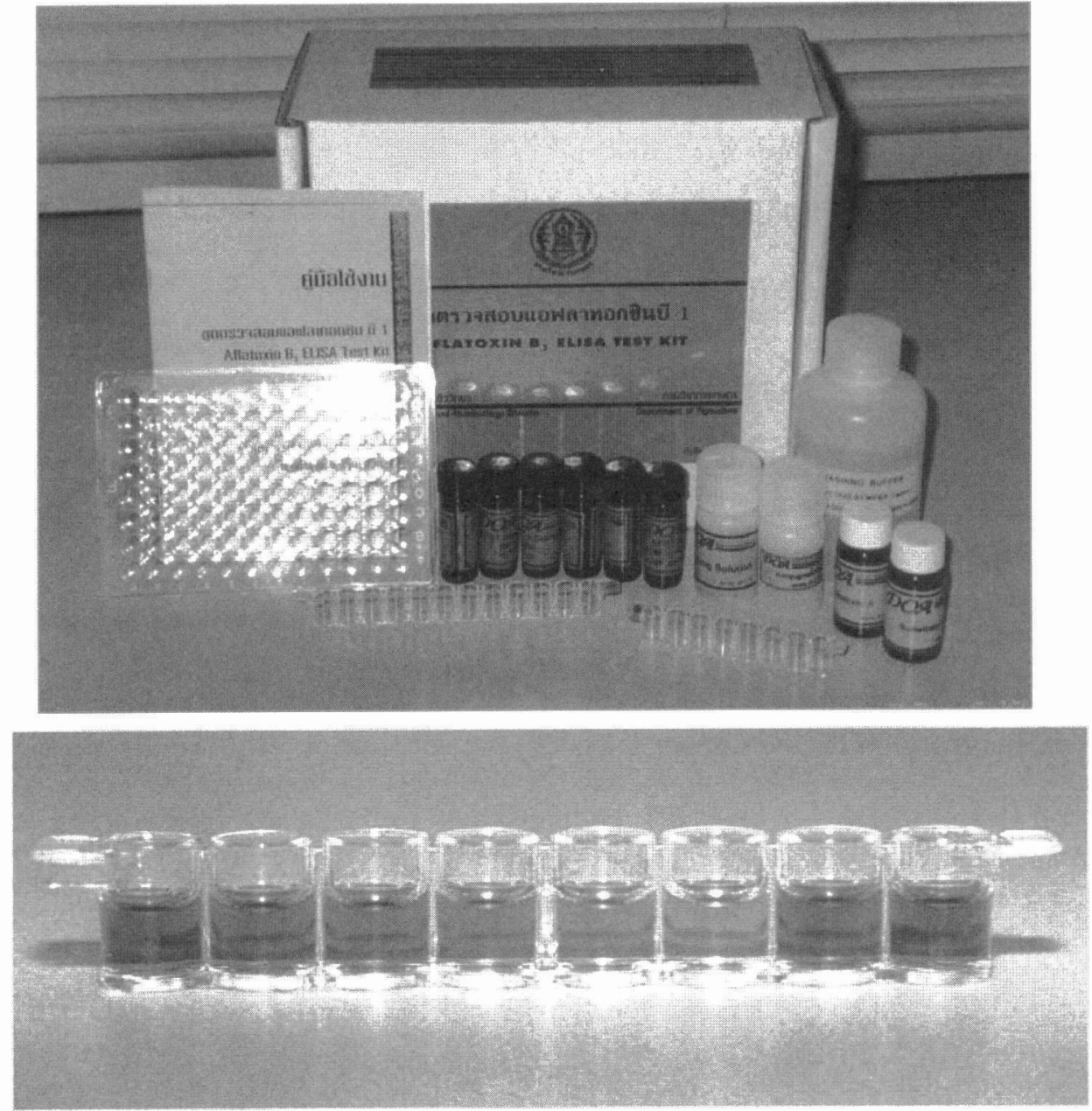

DOA-Aflatoxin ELISA Test Kit, an in-house test kit produced by Department of Agriculture. Ministry of Agriculture and Cooperative. Thailand. 